\title{
LOS CONCEPTOS DE POBREZA, EXCLUSIÓN Y DESIGUALDAD EN LOS ESTUDIOS DE LA ESTRUCTURA SOCIAL ARGENTINA ENTRE 1990 Y 2015
}

\author{
The concepts of poverty, exclusion and inequality in the studies from \\ Argentinian social structures between 1990 and 2015
}

Luis D. Chao*

\section{Resumen}

El artículo propone una revisión de la forma en que los estudios sobre estructura social en Argentina forjaron tres conceptos como herramientas de lectura: pobreza, exclusión y desigualdad. El objetivo de este texto es presentar el planteamiento del problema en un conjunto de análisis que abordaron estas nociones entre 1990 y 2015. Partimos de una definición general de cada uno para luego dar cuenta de los estudios que los han desarrollado en forma particular. Finalmente presentamos la bibliografía organizada a partir de los tres conceptos.

$$
<\text { pobreza }><\text { exclusión }><\text { desigualdad }>
$$

\section{Abstract}

The article propose a revision to the form at the studies about de social structures in Argentina forged three concept like lecture tools: poverty, exclusion and inequality. The objective of this paper is account the approach the problem in a series of analyzes that addressed this notions between 1990 and 2015. We start with a general definition of each and then give an account of the studies that have been developed in particular. Finally, we present the literature organized from the three concepts.

$$
<\text { poverty }><\text { exclusion }><\text { inequality }>
$$

Recibido: 31/01/2016 // Aceptado: 14/03/2016

\footnotetext{
* Licenciado en Comunicación Social. Especialista en Historia Regional. Becario Doctoral- CONICET. 1.daniel.chao@gmail.com
} 


\section{Introducción}

El siguiente trabajo pretende ser una revisión de los principales aportes para el estudio de la estructura social argentina entre 1990 y 2015 a partir de los conceptos de pobreza, exclusión y desigualdad. La propuesta es presentar el modo en que cada uno de estos conceptos fue abordado por diferentes cientistas sociales desde cuatro grandes líneas temáticas: lo económico-laboral, la preocupación por los instrumentos de medición, lo político en la relación Estado-agentes, y lo territorial. El trazado de estas líneas está ligado a la lectura del conjunto de textos que abordamos y supone una de las principales apuestas del escrito.

Este trabajo se centrará en los planteos y problemáticas presentes en un conjunto de análisis sobre la cuestión social, las políticas públicas y los distintos sectores de la estructura social. El texto se divide en tres apartados que inician con una breve definición general de cada concepto para luego dar cuenta de los distintos abordajes y sus principales problematizaciones. Finalmente, proponemos la organización de la bibliografía referenciándola por concepto, con el objetivo de simplificar la búsqueda a otros investigadores interesados en la materia.

\section{Los estudios sobre pobreza}

Los estudios enfocados en la noción de pobreza proliferaron desde la década de los 80' centrados en la distribución del ingreso. El concepto fue trabajado especialmente por la CEPAL (Comisión Económica para América Latina y el Caribe) y apuntó a la dicotomía pobre/no pobre a partir del trazo de una determinada línea diferencial y un conjunto de necesidades básicas insatisfechas. Para algunos autores (Gutiérrez, 2005; Lo Vuolo, 1999), el concepto de pobreza es descriptivo y se centra en identificar mediante ciertos indicadores (como el Índice de Desarrollo Humano) un número finito de carencias. Fue utilizado por numerosos organismos internacionales (Programa de las Naciones Unidas para el Desarrollo [PNUD] o el Banco Mundial) para "medir" Latinoamérica, y sus principales críticas señalan las limitaciones de un solo enfoque (el ingreso) a un solo sector (el pobre) sin percibir otras variables. Este sesgo economicista del concepto mostró sus limitaciones y la necesidad de destacar el carácter multidimensional de la pobreza y su dinámica de producción (Kessler, 2014), la aparición de formas de pobreza distintas y polivalentes (Kessler y Di Virgilio, 2008), y la utilidad de darle al término un carácter explicativo antes que descriptivo.

En Argentina como en el resto del continente estos estudios pusieron el foco en el ingreso y el mercado laboral. Ejemplo de ello es el libro de Luis Beccaria (1990) sobre la precarización del empleo, donde intenta presentar de manera interdisciplinar la relación entre condiciones de trabajo y pobreza. De esta compilación destacamos el trabajo de Beccaria y Orsatti, ya que la pregunta que lo sostiene tiene que ver con precariedad laboral y la estructura productiva entre 1974 y 1988. Es decir, cómo pensar juntos las tasas de desempleo, el tipo de empleo y el modelo productivo de esos 14 años. La pobreza está asociada directamente a calidad de trabajo, apuntando particularmente 
al sector urbano. Esa línea de trabajo es sostenida en tiempo por Beccaria, aunque modificando algunas problemáticas.

Elocuente es el texto presentado junto a Oscar Altimir (Altimir y Beccaria, 2001) que vincula las reformas macroeconómicas (coyunturales y estructurales) y la distribución del ingreso. Los autores marcan una evolución de la pobreza entre 1974 y 1996, cruzando la variable intensidad de la pobreza asociada al acceso y calidad del mercado de trabajo durante el régimen de liberalización del comercio. El nivel descriptivo exhaustivo de este tipo de trabajo se mantiene en abordajes posteriores (Groisman y Beccaria, 2007; Beccaria, Groisman yMonsalvo, 2006) donde retoman la precarización laboral pero a nivel de informalidad. Apuntan a dilucidar con qué intensidad afecta la informalidad al fenómeno de la pobreza argentina entre 1993 y 2005, apelando a ejercicios de simulación y enfocándose en los hogares. Asimismo, ponen en dudas algunos juicios de sentido común, afirmando que no necesariamente informalidad es sinónimo de más pobreza sino que son manifestaciones de un mercado de trabajo que no da respuestas (Beccaria y Maurizio, 2005).

En la misma línea pero apelando a la construcción de un problema diferente, se encuentran los análisis que si bien se estructuran desde la relación mercado laboralmacroeconomía-política, tratan de complejizar el abordaje. En Salvia (2004), por ejemplo, el planteo parte de que la demanda de empleo y sus efectos sobre la desigualdad no es una función directa de las políticas macroeconómicas institucionales, ya que sobre estas operan factores estructurales que remiten al régimen dominante de acumulación y al modo en que los agentes ajustan y despliegan sus estrategias, condicionados por factores ajenos a su voluntad. Un cambio de reglas no es suficiente para el fenómeno heterogéneo de la pobreza, por lo cual el problema es cómo abordar el incremento de empleo con reproducción de la pobreza estructural (inserción laboral, segmentación de los mercados de trabajo y reproducción de sectores marginados del modelo de acumulación). Una propuesta similar por sus grados de cautela respecto a la complejidad de la pobreza, la distribución del ingreso y el mercado de trabajo se encuentra en Lindenboim, Kennedy y Graña (2010) que abordan la noción de distribución funcional del ingreso y su relación con los procesos de reproducción, es decir la lectura al mismo tiempo del tipo de distribución y la participación asalariada en el ingreso como un modo de entender la forma en que se usó la fuerza de trabajo desde inicios de los 90' hasta 2007. El enfoque apunta a la relación entre salario real y productividad; a la par de una revisión de la masa salarial y el consumo, con el objetivo de entender claramente el panorama distributivo y las posiciones de los asalariados frente a la línea de pobreza.

Cabe destacar que existen numerosos trabajos cuya preocupación constituyen un instrumento de lectura de pobreza, más allá de las posibilidades de análisis que permitan. En esa línea pueden destacarse numerosos trabajos de la CEDLAS (Centro de Estudios Distributivos, Laborales y Sociales - UNLP) entre los que podemos señalar los abordajes de Gustavo Busso y Pablo Gluzmann (Busso y Gluzmann, 2012), así como también el trabajo de Busso, Cerimedo, Cicowiez (2005), cuyo propósito es entender el incremento de la tasa de pobreza argentina entre 1992 y 2002 desde la 
noción de movimiento desigualador de distribución del ingreso, dividiendo la lectura en subperíodos y regiones para explicar los factores de incremento de la pobreza. La propuesta es la metodología de descomposición de Mahmoudi, que se centra en la relación entre función de distribución, cambios de ingreso y efecto distributivo. Otros trabajos sostenidos por el problema de la medición son los de Casanova $(2006,2008)$ sobre la noción de trampas de pobreza (caída en la pobreza por breves período de tiempo) en relación a la linealidad de los ingresos. Su propuesta es realizar el seguimiento por hogares a partir de la noción de cohorte y la técnica del pseudo panel. De modo similar señalamos a Conconi y Ham (2007), que se preocupan por definir y operativizar una noción amplia de pobreza a partir de la idea de la construcción de medidas de pobreza multidimensionales; o Alejo y Garganta (2007), cuyo problema está enfocado en construir paneles que permitan ver la rotación de la Encuesta Permanente por Hogar para descomponer la pobreza en dos: crónica y transitoria.

Respecto a abordajes no economicistas resaltamos los que apuntan a las trayectorias de los grupos empobrecidos y sus relaciones con las condiciones de pobreza. Un trabajo importante en este sentido es el de Alicia Gutiérrez (2007) que entiende al fenómeno de la pobreza como un proceso histórico particular enlazado en las dinámicas sociales signadas por el proceso de empobrecimiento progresivo. El problema central es reflexionar sobre estrategias de reproducción de ciertos grupos partiendo del supuesto de que no son independientes de las condiciones objetivas y simbólicas del espacio social global. En este sentido la concepción descriptiva de la pobreza no permite ver causas y relaciones entre ricos y pobres. En base a este planteo, sus decisiones metodológicas están ligadas al análisis relacional para los estudios de pobreza, abordando cuestiones como los recursos de la pobreza y capital social y el concepto de redes sociales que le permite ver una articulación entre pobres y ricos. Por un camino similar encontramos los trabajos de Amalia Eguía (Eguía, 2004; Eguía y Ortale, 2004), centrados en las estrategias familiares de reproducción para el estudio de las condiciones de vida. En líneas generales el problema parte de una noción de pobreza multidimensional y heterogénea, por lo cual, el análisis considera dimensiones vinculadas a los hogares, la organización doméstica, los recursos de reproducción, la participación comunitaria en la política, es decir las estrategias familiares; y la lectura tensiona los recursos con la situación estructural, configuradas de acuerdo al universo de significados de los sujetos. En la misma línea de la pregunta por lo político en torno a la pobreza, pero centrado en las políticas públicas, ubicamos los trabajos de Rubén Lo Vuolo (1998; 1996) especialmente el libro La pobreza... de la política contra la pobreza (Lo Vuolo y otros, 1999), donde la pobreza es tratada en términos de inserción y cohesión, y de allí presenta una lectura posible de las políticas sociales que breguen por construir redes de seguridad social. El texto se erige diagnóstico proponiendo indicadores de lectura del fenómeno y de los tipos de acercamiento por parte del Estado argentino, particularmente en la década de los 90'. Un enfoque sugestivo al respecto es el de Emilio Ayos (2012; 2010) quien plantea una intersección entre pobreza y delito a partir de las políticas que los abordan. Para el autor existe una imbricación entre pobreza y delito y las formas 
de gestión de ambos entre 2003-2008, que tiene que ver con las maneras en que se producen y entrelazan a partir de la construcción de una clase peligrosa.

Por último, nos centramos en aquellos trabajos que dan cuenta de la relación entre territorio y pobreza, como los de Álvarez, Gómez, Lucarini y Olmos (2007) sobre comportamiento demográfico en Argentina. Allí el problema es la posibilidad de medir la pobreza a partir de las estrategias demográficas diferenciales según condición de privación y las diferencias geográficas. En esa línea podemos situar a Kessler y De Virgilio (2008) sobre la "nueva pobreza", las estrategias de los hogares en contexto de pauperización y las diferencias geográficas en las dimensiones del empobrecimiento y las posibilidades de hacerle frente a partir de una serie de recursos (como el capital social).

\section{Abordajes sobre la exclusión social}

El uso del término exclusión se vincula a un intento de ampliar el campo de mirada sobre la estructura social. Ligado a la Organización Internacional del Trabajo (OIT), se concentró en sus inicios hacia el mercado laboral (influenciado por el trabajo de Robert Castel) y luego hacia la privación de otras necesidades (tierra, justicia), las cuales pueden generar situaciones de indefensión (Kessler, 2014). La preocupación general apunta a pensar cómo el sistema social genera procesos que excluyen individuos o grupos en un momento y lugar, por lo cual la reflexión sobre la medición cobra un sentido menos descriptivo que en la pregunta por la pobreza (Busso, 2005).

Existen un conjunto de estudios que ligan la exclusión con el análisis del mercado de trabajo como espacio socializador por excelencia, por ende principal causante de fragmentación. Por ejemplo Lindenboim, Serino y González (2000; Lindenboim y González, 2004) definen la precarización de las relaciones laborales como una forma de exclusión. El punto nodal en estos trabajo es entender a la precarización como un modo de reproducción de la relaciones entre trabajadores y empleadores y otras categorías sociales, en el marco de una imposibilidad de integración y respuesta del Estado. En esa misma línea, pero apuntando a las estrategias de reproducción de los hogares, Agustín Salvia (2007; 2002) mantiene la idea de entender al fenómeno de la exclusión como estructurado fundamentalmente en la fragmentación de las relaciones laborales; sin embargo el problema se estructura en cómo se dan las estrategias reproductivas en hogares desprotegidos y los impactos de las condiciones estructurales. Una de las nociones fundamentales que ligan estos estudios es el de estructura de oportunidades para pensar la línea entre inclusión/exclusión con el objetivo de abordar las trayectorias laborales de los jóvenes trabajadores. En coincidencia, Gonzalo Saraví (2004; 2001) se pregunta sobre el impacto de los procesos de exclusión laboral en las biografías laborales y experiencias de la juventud. La hipótesis central es que las condiciones de desventajas estructurales, más la nuevas desventajas para la inclusión de los 90, impacta en la posibilidad de transición a la adultez y su integración plena. En ese sentido María Cristina Bayón (2008; 2006) toma el enfoque biográfico para abordar el proceso de exclusión a partir de la articulación de una lectura de macroprocesos con microprocesos 
y microtransformaciones. El objeto para la autora son las familias y la pregunta inicial es el impacto diferencial sobre las identidades, experiencias y perspectivas de futuro en la última década del siglo XX en Argentina. Señalamos también una serie de trabajos que apuntan a pensar instrumentos eficaces de lectura de la exclusión. En ese sentido remarcamos los trabajos de Alberto Minujin (1996; Minujin y López, 1994) que parten del acceso a diferentes bienes y la participación asalariada para comprender el grado de desintegración de la sociedad argentina. Minujin resalta el lugar de la distribución del ingreso para dar cuenta de la fragmentación de la sociedad y el papel de las políticas públicas en ese proceso. Con un objetivo similar, pero proponiendo lectura de casos en contextos de exclusión, Pablo Forni (2005; Forni, Siles y Barreiro, 2004) presenta una serie de estudios que dan cuenta de las formas en que el capital social adquirido puede modificar las estrategias de los hogares de barrios pobres.

A diferencia de los otros conceptos, el de exclusión dio paso a una serie de trabajos sobre identidad y cohesión entendida como un modo de reconocer al otro. En ese sentido, Maristella Svampa $(2005 ; 2000)$-a partir de lo que llama la modernidad excluyente- se pregunta por el tipo de exclusión que atraviesa a la Argentina desde inicios de la década de los 90. Propone una perspectiva política para entender la estructura social y su tipo de fragmentación tanto material como simbólica. Para la autora, existe un tipo de práctica de exclusión por parte del Estado que cruza las relaciones de trabajo, de espacio y de territorio. En una clave similar, señalamos los trabajos de Elizabeth Jelin $(2001 ; 1994)$ respecto a la noción de exclusión y sus características en nuestro país. Para la autora, lo que permite el concepto es entender la pobreza en clave política y social antes que económica, por lo cual su problema se centra en las condiciones de ciudadanía en contexto de desventajas estructurales.

Existen además una serie de estudios volcados a comprender el fenómeno de exclusión desde su inscripción en el territorio. Podemos destacar algunos trabajos de Fernando Díaz Orueta (Díaz Orueta y Seoane, 2003; Díaz Orueta, Seoane y Agulles Martos, 2001) en los cuales la pregunta pasa por pensar la exclusión en territorios donde no se expresa tan claramente. La fragmentación es la incapacidad de incorporación de grupos de individuos a la ciudad en tanto que sujetos políticos, complejizando el término para reflexionar sobre la idea de inclusión/exclusión desde otra perspectiva. Lo que se manifiesta en Buenos Aires es una recualificación de su organización espacial, distribuyendo la exclusión en zonas poco claras disminuyendo la capacidad de articulación políticas de grupos de individuos. Con un enfoque distinto, podemos señalar los trabajos de Carlos Fidel $(2010 ; 2008)$ que intenta operativizar en conjunto la noción de exclusión y condición de vida para leer un territorio en particular (el Partido de Quilmes - Buenos Aires), con el objetivo de describir el estado de situación a nivel micro con una densidad empírica fuerte. Así también podemos mencionar los trabajos de Elma Montaña $(2013 ; 2007)$ que plantea la noción de exclusión pero desde un punto de vista que unifique espacio-sujetos, marcando los procesos regionales de fragmentación territorial. El problema para la autora es cómo pensar el proceso productivo en relación a la segregación territorial en las provincias, que configuran espacios olvidados donde se apoyan identidades excluidas y excluyentes. 


\section{Los estudios sobre desigualdad}

El abordaje de la estructura social en términos de desigualdad parte de las críticas sobre el concepto de exclusión y su uso académico señalado como vago y con capacidad descriptiva limitada. Kessler (2014) afirma que las debacles económicas y sociales latinoamericanas desplazaron la pregunta por la exclusión hacia la desigualdad lo que, a su criterio, amplía los estudios previos. La noción permite una mirada diferencial en múltiples esferas, por lo cual cada lectura particular puede arrojar resultados que permitan tomar una fotografía más amplia y pensar en cuestiones como el acceso (poniendo a los sujetos en un lugar activo de lectura) antes que en la carencia.

Respecto a los estudios que ligan la desigualdad con la distribución del ingreso podemos destacar a Altimir, Beccaria y Rozada (2002; 2001) quienes se centran en la distribución del ingreso familiar desde diversas esferas (entre hogares, entre ingresos, entre ocupaciones) tomando como base el crecimiento del Gini. Esos trabajos cruzan ingreso, pobreza, mercado de trabajo e impacto del mismo en el ingreso familiar, abordándolos por ejercicios de microsimulación en una línea evolutiva que inicia desde mediados de los 70 hasta el 2000. En los mismos términos, Beccaria y Groisman (2006; 2005) construyen su problema cruzando el comportamiento macroeconómico argentino entre 1975-2007 con los rasgos de la realidad laboral y los ingresos. Su abordaje entrecruza características políticas redistributivas con condiciones de desigualdad. Sobre la misma base de desigualdad en el ingreso, pero con el objetivo de hacer operativos ambos términos para un análisis en diferentes regiones, aparecen los trabajos de Beccaria, Esquivel y Maurizio (2005; 2002). En primer término plantean el abordaje de la desigualdad (dispersión de las diferencia) en paralelo con el de la polarización (posibilidad de homogeneizar grupos en el análisis y medir sus distancias), y luego analizan las características macroeconómicas vinculadas a la convertibilidad y su salida a fines de los 90’ en relación a la distribución del ingreso.

El siguiente grupo de trabajos propone un estudio sobre la desigualdad que pueda articularse con la distribución del ingreso, pero que a su vez amplíe la mirada. En este sentido, podemos decir que son trabajos que apelan a la visión multidimensional de la desigualdad promoviendo el análisis de diversos factores. Gasparini, Escudero y Marcchioni (2001) plantean complejizar el análisis de la distribución del ingreso, cruzando los términos pobreza y desigualdad. El problema central del texto es cómo articular un abordaje de la desigualdad de ingreso con otros factores (lo cotidiano, lo político) como condiciones de emergencia de elementos que moldean el tipo de distribución. Profundizando en factores económicos y políticos, y avanzando en el trabajo empírico, Cruces y Gasparini (2008; 2009) se preguntan sobre los patrones de desigualdad en argentina desde la década de los 70. Así plantean exceder la distribución del ingreso, presentando regularidades a partir de tres hipótesis sobre la desigualdad (cruzada por la distinción de períodos): liberalizacion comercial, cambio tecnológico y ajuste estructural. A su vez, Kessler (2014) propone la multidimensionalidad para complejizar la lectura de las desigualdades en un contexto de crecimiento económico, por lo cual presenta una hipótesis de desigualdad multifacética y con tendencias 
contrapuestas. Su propuesta es abordar tanto individuos, como clases o grupos con regularidades distintas a partir de diversas dimensiones o esferas de bienestar (ingreso, educación, salud, vivienda, delito, territorio, infraestructura, etc.) desde la noción de igualdad de posiciones, es decir una forma de leer la relación estructura-sujetos desde el conjunto de beneficios más o menos similares que éstos pueden alcanzar.

Respecto a la cuestión de las desigualdades territoriales, podríamos diferenciar dos enfoques. Por un lado aquellos que hacen hincapié en las distancias entre regiones del país, y por otro, los estudios que marcan al espacio urbano como un terreno donde se visibilizan las desigualdades. Para el primero de los enfoques, señalamos los trabajos de Josefina Vaca y Horacio Cao (Cao y Vaca, 2005; 2004) quienes abordan el desarrollo desigual (político, cultural, económico) entre las regiones argentinas desde una perspectiva histórica, a partir de la pregunta por la "excepcionalidad" de los 80 y 90. La base es el convencimiento de los autores de que la asimetría regional -y la planificación histórica de un fuerte centralismo- posiciona al país como periférico en el concierto mundial y que la heterogeneidad y reproducción de las desigualdades son fundamentales para comprender la estructura social. Por su parte, con un horizonte similar, pero apelando a un enfoque economicista, los estudios de Fernando Gatto y Oscar Centrángolo (Gatto y Centrángolo, 2007; Steimberg, Gatto y Centrángolo, 2011; Gatto, 2007) plantean el problema de la desigualdad provincial a partir de la relación entre respuestas económicas y competitividad con la estructura productiva. La mirada sobre la desigualdad está en perspectiva institucional, es decir sobre el desarrollo de los aparatos privados y públicos para crear condiciones de desarrollo.

Por otro lado, los enfoques que hacen una lectura urbana de la desigualdad (en su gran mayoría centrados en la Ciudad de Buenos Aires) a su vez podrían subdividirse en dos. Aquellos que entienden la organización territorial y la segmentación urbana como una forma de reproducir la desigualdad. En esta línea incluimos los trabajos de Prévôt Schapira $(2014 ; 2002)$ y de Groisman $(2009 ; 2010)$ que reflexionan sobre el tejido urbano y la fragmentación en relación a aspectos socioeconómicos. En la segunda subdivisión se encuentran los trabajo que entienden la desigualdad en relación a las formas en que las personas intentan acceder a la ciudad, es decir los modos circulación y tránsito, la migraciones internas, etc. Los estudios de Daniela Soldano $(2013 ; 2010)$ para el caso argentino son elocuentes, ya que la autora estructura sus preguntas en torno a la vida cotidiana. Recupera la mirada sobre la fragmentación y la pone en relación a la movilidad, para afirmar que el Estado reproduce relaciones desiguales que se manifiestan material y simbólicamente, por la cual se tensiona la relación espaciosubjetividad. Por último, cabe destacar algunos trabajos que abordan la cuestión de la desigualdad y la política. Los aportes de Javier Auyero (2012; 2004) son fundamentales, ya que el autor equipara desigualdad política con desigualdad social, y construye su problema en términos de acaparamiento, clientelismo y líneas de desigualdad barrial por parte de referentes del Partido Justicialista. El abordaje interpela las prácticas de la vida cotidiana, el desplazamiento y monopolio de recursos de bienestar y sobre todo las reciprocidades como modos de reproducir las desigualdades. 


\section{A modo de cierre}

Nuestro trabajo abordó los conceptos de pobreza, desigualdad y exclusión mediante una revisión de los principales aportes para el estudio de la estructura social argentina. La propuesta fue pensar estos conceptos articulados en grandes líneas temáticas con ciertas regularidades: lo económico-laboral, la preocupación por el instrumento de medición, lo político tensionando la relación Estado-agentes y lo territorial. Pudimos ver que, si bien estos temas cruzan casi todos los estudios, ciertos conceptos hacen mayor hincapié en alguno de estos puntos (p.e. la ligazón pobreza con sus instrumentos de medición, la desigualdad con lo económico-laboral y la exclusión con el papel del Estado). En síntesis, este estado de la cuestión intentó una recuperación cuyo norte fue construir una base documental teórica sobre las formas en que la cuestión social fue pensada, las políticas sociales fueron desarrolladas, y las categorías sociales objeto de esas políticas fueron teorizadas en los últimos veinticinco años.

\section{Referencias bibliográficas}

Pobreza

Alejo, J.; Garganta, S. 2014. Pobreza Crónica y Transitoria: Evidencia para Argentina 1997-2012. La Plata, CEDLAS, vol 175.

Álvarez, G.; Gómez, A.; Olmos, M. 2007. "Pobreza y comportamiento demográfico en Argentina: La heterogeneidad de la privación y sus manifestaciones". En: Papeles de población, México, Universidad Autónoma de México, N 51, Vol. 13, p. 77-110.

Ayos, E. 2010. "Individualización y prevención" social” del delito en Argentina: la reactualización de las clases peligrosas". En: Revista Katálysis, Florianópolis, UFSC, $\mathrm{N}^{\circ} 13$, Vol. 2, p. 220-228.

Ayos, E. 2012. "Prevención del delito y políticas sociales en Argentina: tres ejes problemáticos". En Revista de ciencias sociales, Costa Rica, UCR, N 135-136, p. 113-126.

Beccaria, L. (Comp). 1990. La precarización del empleo en la Argentina, Buenos Aires, Centro Editor de América Latina.

Beccaria, L.; Groisman, F.; Monsalvo, P. 2006. "Segmentación del mercado de trabajo y pobreza en Argentina”. En: XLI Reunión Anual de la Asociación Argentina de Economía Política, Universidad Nacional de Salta, Noviembre 15-16, Salta.

Beccaria, L.; Maurizio, R. 2005. Mercado de Trabajo y Equidad. Buenos Aires, Prometeo.

Beccaria, L.; Minujin, A. 1991. "Sobre la medición de la pobreza: enseñanzas a partir de la experiencia argentina". En: Documentos de trabajo, Buenos Aires, UNICEF, $\mathrm{N}^{\circ} 8$, p.39

Casanova, L. 2006. “Análisis estático y dinámico de la pobreza en Argentina: Evidencia empírica para el período 1998-2002”. En: Documentos de Trabajo del CEDLAS, La Plata, CEDLAS-UNLP, N`31. 
Casanova, L. 2008. "Trampas de pobreza en Argentina: evidencia empírica a partir de un pseudo panel”. En: Documentos de trabajo del CEDLAS, La Plata, CEDLASUNLP, $\mathrm{N}^{\circ} 64$.

Cerimedo, F.; Cicowiez, M. 2005. "Pobreza, crecimiento y desigualdad: Descifrando la última década en Argentina”. En: Documentos de Trabajo del CEDLAS, La Plata, CEDLAS-UNLP, N²1.

Conconi, A.; Ham González, A. 2007. "Pobreza multidimensional relativa: Una aplicación a la Argentina". En: Documentos de Trabajo del CEDLAS, La Plata, CEDLAS-UNLP, N57.

Eguía, A. 2004. "Pobreza y reproducción familiar: propuesta de un enfoque para su estudio". En: Caderno CRH, Bahía, UFB, N40, Vol.17, pp. 79 - 92.

Gutiérrez, A. 2005. Pobre, como siempre: estrategias de reproducción social en la pobreza, Córdoba, Ferreyra Editores, p. 453.

Kessler, G.; Di Virgilio, M. 2008. “La nueva pobreza urbana: dinámica global, regional y argentina en las últimas dos décadas". En: Revista de la CEPAL, Naciones Unidas, CEPAL, N95, pp. 31-50.

Lindenboim, J.; Kennedy, D.; Graña, J. 2010. "El debate sobre la distribución funcional del ingreso”. En: Desarrollo Económico, Buenos Aires, IDES, N¹96, Vol49, pp. 541-571.

Lo Vuolo, R. (Comp.). 1999. La pobreza... de la política contra la pobreza, Buenos Aires, Miño y Dávila.

Salvia, A. 2005. "Crisis del empleo y nueva marginalidad: el papel de las economías de la pobreza en tiempos de cambio social." En: Los Nuevos Rostros de la Marginalidad, Buenos Aires, Editorial BIBLOS.

\section{Desigualdad}

Altimir, O.; Beccaria, L. 2001. "El persistente deterioro de la distribución del ingreso en la Argentina". En: Desarrollo Económico, Buenos Aires, IDES, N $16^{\circ}$, Vol. 40, pp. 589-618.

Altimir, O.; Beccaria, L.; González Rozada, M. 2001. "La evolución de la distribución del ingreso familiar en Argentina". En: Serie de Estudios en Finanzas Públicas, La Plata, SEDICI.

Auyero, J. 2004. Clientelismo político. Las caras ocultas, Buenos Aires, Capital intelectual.

Auyero, J. 2012. La política de los pobres: Las prácticas clientelistas del peronismo, Buenos Aires, Ediciones Manantial.

Beccaria, L.; Groisman, F. 2006. "Inestabilidad, movilidad y distribución del ingreso en Argentina”. En: Revista de la CEPAL, Naciones Unidas, CEPAL, N89, pp. 133-156.

Beccaria, L.; Esquivel, V.; Maurizio, R. 2005. "Empleo, salarios y equidad durante la recuperación reciente en la Argentina”. En: Desarrollo Económico, Buenos Aires, IDES, N¹78, Vol. 45, pp. 235-262. 
Cao, H.; Vaca, J. 2005. "Continuidades y rupturas en las desigualdades territoriales de la República Argentina”. En: Revista de estudios regionales, Málaga, UPA, Nº72, pp. 141-164.

Cao, H.; Vaca, J. 2004. "La división regional del trabajo en la Argentina: nuevos elementos y tradicionales desequilibrios". En: Realidad Económica, Buenos Aires, IADE, N²02, pp. 65-86.

Cruces, G.; Gasparini, L. 2009. "Desigualdad en Argentina. Una revisión de la evidencia empírica Segunda Parte". En: Desarrollo Económico, Buenos Aires, IDES, N 193, Vol. 49, pp. 3-29.

Gasparini, L.; Cruces, G. 2008. "Una distribución en movimiento: El caso de Argentina”. En: Documento de Trabajo del CEDLAS, La Plata, CEDLAS-UNLP, $\mathrm{N}^{\circ} 78$.

Gatto, F. 2007. “Crecimiento económico y desigualdades territoriales: algunos límites estructurales para lograr una mayor equidad”. En Kosacoff, B. (ed.). Crisis, recuperación y nuevos dilemas. La economía argentina 2002-2007, Buenos Aires, CEPAL.

Gatto, F.; Cetrángolo, O. 2003. "Dinámica productiva provincial a fines de los años noventa". En: Estudios y perspectivas, Buenos Aires, CEPAL, No 14.

Groisman, F. 2009. "Segregación residencial socioeconómica en Argentina durante la recuperación económica (2002-2007)". En: Documento de Trabajo IELAT, Madrid, U. de Alcalá, $\mathrm{N}^{\circ} 03$.

Kessler, G. 2014. Controversias sobre la desigualdad: Argentina 2003 - 2013, Buenos Aires, Fondo de Cultura Económica.

Prévôt Schapira, M. F. 2002. “Buenos Aires en los años 90’: metropolización y desigualdades”. En: Revista EURE, Santiago, PUCC, N85, Vol²8, pp. 31-50.

Prévôt Schapira, M. F. 2014. "Segregación, fragmentación, secesión. Hacia una nueva geografía social en la aglomeración de Buenos Aires". En Revista Economía, Sociedad y Territorio, México, El Colegio Mexiquense, N 7, Vol. 2.

Soldano, D. 2013. "Confinamientos, movilidad e intercambios. Una investigación sobre las condiciones y los modos de vida en la periferia del Gran Buenos Aires".En: Neiva Vieira da Cunha, M.; Segura, R. (Coord). Segregación y diferencia en la ciudad, Quito, FLACSO.

Soldano, D. 2010. "Espacios comunes, sociabilidad y Estado. Aportes para pensar los procesos culturales metropolitanos". En: Apuntes del CECYP, Buenos Aires, Fundación del Sur, $\mathrm{N}^{\mathrm{0}}$ 17, pp. 79-96.

Steinberg, C.; Gatto, F.; Cetrángolo, O. 2011. "Desigualdades territoriales en la Argentina: insumos para el planeamiento estratégico del sector educativo". En: Documentos de Proyectos, Naciones Unidas, CEPAL, N³91.

\section{Exclusión}

Bayón, M. 2006. "Precariedad social en México y Argentina”. En: Revista de la CEPAL, Naciones Unidas, CEPAL, N 88, pp. 133-152. 
Bayón, M. 2008. "Desigualdad y procesos de exclusión social. Concentración socioespacial de desventajas en el Gran Buenos Aires y la Ciudad de México". En: Estudios demográficos y urbanos, México, Colegio de México, $\mathrm{N}^{\circ} 1$, Vol.23, pp. 123-150.

Díaz Orueta, F.; Lourés Seoane, M.; Agulles Martos, J. 2001. "Ciudad, democracia y movimientos sociales: el Movimiento de Ocupantes e Inquilinos (MOI) de Buenos Aires". En: VIII Encuentro de Latinoamericanistas Españoles, Consejo Español de Estudios Iberoamericanos, Noviembre 13-15, Madrid.

Díaz Orueta, F.; Lourés Seoane, M. 2003. "La ciudad postfordista: economía cultural y recualificación urbana”. En Revista de Economía Crítica, Madrid, ACEC, N², pp. 105-121.

Fidel, C.; Di Tomaso, R.; Farías, C. 2008. Territorio, condiciones de vida y exclusión: el partido de Quilmes (Provincia de Buenos Aires, Argentina). Buenos Aires, CLACSO.

Forni, P.; Siles, M.; Barreiro, L. 2004. “¿Qué es el Capital Social y cómo Analizarlo en contextos de Exclusión Social y Pobreza?”. En: Research Report, East Lansing, JSRI, $\mathrm{N}^{\circ} 35$.

Forni, P. 2005. "Organizaciones comunitarias y redes sociales: sus implicancias en la generación de capital social y la superación de situaciones de exclusión. Estudios de caso en Buenos Aires". En: III Seminario para el análisis de redes sociales en Sevilla, RHARS, Noviembre 7-9, Sevilla.

Jelin, E. 1994. “¿Ciudadanía emergente o exclusión? Movimientos sociales y ONGs en los años noventa". En: Revista mexicana de sociología, México, UNAM, $\mathrm{N}^{\circ} 4$, Vol, 56, pp. 91-108.

Jelin, E. 2001 Exclusión, memorias y luchas políticas. Buenos Aires, CLACSO.

Lindenboim, J.; Serino, L.; González, M. 2000. "La precariedad como forma de exclusión". En Cuadernos del CEPED, Buenos Aires, UBA, ํㅜ 4 .

Lindenboim, J.; González, M. 2004. "El neoliberalismo al rojo vivo: mercado de trabajo en Argentina". En: Cuaderno del CEPED, Buenos Aires, UBA, Nº , pp. 27-48.

Montaña, E. 2007. "Identidad regional y construcción del territorio en Mendoza (Argentina): memorias y olvidos estratégicos". En: Bulletin de l'Institutfrançaisd'étudesandines, CNRS, N³6, Vol.2, pp. 277-297.

Montaña, E. 2013. "Las disputas territoriales de una sociedad hídrica. Conflictos en torno al agua en Mendoza, Argentina". En: Revista Iberoamericana de Economía Ecológica, México, CIEC-UNAM, Vol'9, pp. 1-17.

Minujin, A. 1996. Desigualdad y exclusión: desafíos para la política social en la Argentina de fin de siglo. Buenos Aires, Unicef/Losada.

Minujin, A.; López, N. 1994. "Nueva pobreza y exclusión: el caso argentino". En: Nueva sociedad, $\mathrm{N}^{\circ} 131$, pp. 88-105.

Salvia, A. 2002. "La estructura social del trabajo en Argentina: desempleo, subempleo y precariedad laboral". En: Documento de Investigación AE, Buenos Aires, UCA, $\mathrm{N}^{\circ} 01$. 
Salvia, A. 2007. "Consideraciones sobre la transición a la modernidad, la exclusión social y la marginalidad económica. Un campo abierto a la investigación social y al debate político". En Salvia, A.; Chávez, M. (Comps.). Sombras de una marginalidad fragmentada. Aproximaciones a la metamorfosis de los sectores populares de la Argentina, Buenos Aires, Miño y Dávila, pp. 25-67.

Saraví, G. 2004. "Segregación urbana y espacio público: los jóvenes en enclaves de pobreza estructural". En: Revista de la CEPAL, Naciones Unidas, CEPAL, $\mathrm{N}^{\circ} 83$, pp. 33-48.

Saraví, G. 2001. "Entre la evasión y la exclusión social: jóvenes que no estudian ni trabajan". En: Nueva Sociedad, $N^{\circ} 190$, pp. 71-85

Svampa, M. 2005. La sociedad excluyente: la Argentina bajo el signo del neoliberalismo, Buenos Aires, Taurus.

Svampa, M. 2000. "Clases medias, cuestión social y nuevos marcos de sociabilidad". En: Punto de vista, Buenos Aires, UNGS, N67, Vol. 23, pp. 34-40. 
